\title{
ISM gas removal from starburst galaxies and the premature death of star clusters
}

\author{
C. Melioli and E. M. de Gouveia Dal Pino
}

Universidade de São Paulo, IAG, Rua do Matão 1226, Cidade Universitária, São Paulo 05508-900, Brazil

e-mail: [cmelioli; dalpino] @astro.iag.usp.br

Received 13 September 2005 / Accepted 21 October 2005

\section{ABSTRACT}

Recent observational studies of the age distribution of star clusters in nearby merging galaxies and starburst (SB) galaxies indicate a premature death of the young clusters. The fate of an evolving star cluster crucially depends of its gas content. This behaves like a glue that helps to keep the star system gravitationally bound. In SB systems where the rate of supernovae ( $\mathrm{SNe}$ ) explosions is elevated one should expect an efficient heating of the gas and its complete removal which could then favor the rapid dissociation of the evolving star clusters. Based on a contemporaneous study of the dynamical evolution of the interstellar gas in SB environments (Melioli \& de Gouveia Dal Pino 2004, A\&A, $424,817)$ where it has been considered also the presence of dense clouds that may inhibit the heating efficiency of the interstellar gas by the $\mathrm{SNe}$, we have here computed the timescales for gas removal from young clusters embedded in these systems and found that they are consistent with the very short timescales for cluster dissolution which are inferred from the observational studies above. Our results indicate that typical SB proto-clusters should start to disperse after less than 5 Myr. For a given total gas mass content, this result is nearly insensitive to the initial star formation efficiency.

Key words. stellar clusters: general - stellar cluster: ISM, SNe

\section{Introduction}

Star clusters in nearby SB galaxies seem to contribute with only $\sim 20 \%$ of the light seen in UV images, so that the diffuse light is dominated by the contribution of field stars (Meurer et al. 1995). There is increasing observational evidence that suggests that this diffuse UV field light is actually due to the dispersion of aged star clusters that have left their remaining stars in the field (Tremonti et al. 2001; Lada \& Lada 2003; Fall 2004). Chandar et al. (2005) have observed this trend in eleven nearby SB galaxies and have also identified a lack of massive stars in the field which supports the interpretation above that the field stars are composed of older, dissolving clusters. They also estimate that the star clusters need to dissolve on timescales 7-10 Myr in order to create the field stars.

A contemporaneous statistical study of the age distribution of star clusters in nearby merging galaxies (the Antannae galaxies) by Fall (2004; see also Whitmore et al. 2005) has shown that the number of clusters decreases by a factor of $\sim 10$ by the time the cluster population has reached the age of $\sim 10 \mathrm{Myr}$. This rapid decline is very insensitive to the cluster mass, at least for masses above $3 \times 10^{4}$ solar masses, and should be an indication that most of the clusters have become gravitationally unbounded.

The fraction of gas mass that is converted into stars in SBs is about 10 times larger than in normal galaxies and, as such, the production of a high number of massive stars results high rates of SN explosions. Thus SN explosions, as well as the ionizing radiation from massive stars, and stellar winds may efficiently heat the gas and eventually remove a significant fraction of the ISM from a proto-cluster, leaving the stars gravitationally unbound. The crossing time for stars orbiting within a bound cluster or proto-cluster is $\tau_{\mathrm{cr}} \simeq 2 R / \sigma$, where $\sigma$ is the velocity dispersion of cluster, $\sigma \simeq\left[0.4 G\left(M_{\mathrm{star}}+M_{\mathrm{gas}}\right) / R\right]^{1 / 2}$, and $R$ is its the radius.

Now, if a proto-cluster suddenly loses most of its mass by the removal of the gas and the instantaneous velocity dispersion of the stars at the time of gas removal, $\sigma$, becomes larger than the escape velocity, $v_{\text {esc }} \simeq\left[\left(2 G M_{\text {star }}\right) / R\right]^{1 / 2}$, then the cluster will be no longer gravitationally bound and will expand almost freely with its radius increasing with age as (see, e.g., Fall 2004; Lada \& Lada 2003) $R_{\tau} \simeq R\left(\tau / \tau_{\mathrm{cr}}\right)$ and its surface density decreasing as $\Sigma_{\tau} \simeq \Sigma\left(\tau / \tau_{\mathrm{cr}}\right)$.

A recent independent theoretical study of the evolution of the ISM of SB environments by Melioli \& de Gouveia Dal Pino (2004; see also Melioli et al. 2005), has revealed that the effectiveness of the SNe and their shock waves to heat the IS gas and make it to be ejected from the SB system is sensitive to the total gas mass content. In particular, we have found that if the total gas mass both in the form of clouds and diffuse gas is large enough, then the radiative cooling time scale of the gas can remain shorter than the timescale for the development of a hot 
Table 1.

\begin{tabular}{cccccc}
\hline \hline Model & $n\left(\mathrm{~cm}^{-3}\right)$ & $T(\mathrm{~K})$ & $R(\mathrm{pc})$ & $M_{\text {gas }}\left(M_{\odot}\right)$ & $M_{\text {star }}\left(M_{\odot}\right)$ \\
\hline 1 & 10 & $10^{4}$ & 10 & $8 \times 10^{6}$ & $10^{6}$ \\
2 & 10 & $10^{4}$ & 20 & $8 \times 10^{6}$ & $10^{6}$ \\
3 & 10 & $10^{4}$ & 5 & $8 \times 10^{5}$ & $10^{5}$ \\
4 & 10 & $10^{4}$ & 10 & $8 \times 10^{5}$ & $10^{5}$ \\
\hline
\end{tabular}

superbubble (due to the SNRs interactions) for about half lifetime of the SB. During this time, most of the gas is retained in the $\mathrm{SB}$, but after this period the SNe heating efficiency rapidly increases to its maximum value thus leading to gas expansion and removal from the SB in a short period. The clouds play a fundamental role in this process as they work like valves that are able to both retain part of the gas to themselves and lose part of it to the diffuse ISM through cloud photoevaporation and then, maintain the ISM density and the radiative cooling rate high enough for a long time. As long as the gas is retained it may favor new generations of star formation.

In this work, we apply the analysis above, which was performed for an entire SB system (Melioli \& de Gouveia Dal Pino 2004), to the scale of individual star proto-clusters embedded in it, and show that the resulting typical timescales both for gas retention and removal from these clusters are consistent with the very short timescales for cluster dissolution inferred from the previous investigations of Fall (2004) and Chandar et al. (2005) of local SB galaxies

\section{A model for the evolution of the gas}

At least two physical parameters are essential to determine the evolution of a proto-cluster: the star formation efficiency and the timescale of gas dispersal from the cluster (e.g., Lada \& Lada 2003).

Accurate measurements of the star formation efficiency, $S F E=M_{\text {star }} /\left(M_{\text {gas }}+M_{\text {star }}\right)$, which require a reliable determination of both the gaseous and the stellar mass content, are in general not available for cluster forming regions, nor even in our galaxy. In the latter, the inferred SFEs of proto-clusters embedded in giant molecular clouds range from $\sim 10 \%$ to $30 \%$.

The timescale for gas removal, $\tau_{\mathrm{g}}$, is even less constrained by empirical data. In SB systems, in particular where the rates of SN explosions are elevated, one should expect an efficient heating of the gas followed by its complete removal from the proto-cluster. The condition for a cluster to become gravitationally unbound after a rapid gas dispersal (e.g., in a timescale $\tau_{\mathrm{g}} \leq \tau_{\mathrm{cr}}$ ) is that the instantaneous velocity dispersion of the stars at the time of gas removal is larger than the escape velocity, or $\sigma>v_{\text {esc }}$. From the equations for $\sigma$ and $v_{\text {esc }}$ given in Sect. 1, one finds that this condition implies that a system with $S F E \leq 50 \%$ should become unbound after rapid gas removal. (e.g., Lada \& Lada 2003). In the opposite case of a slow gas removal $\left(\tau_{\mathrm{g}}<\tau_{\mathrm{cr}}\right)$ even clusters with low SFEs would have time enough to adjust adiabatically and expand to new states of virial equilibrium and remain bound.

However, the simple estimates above, do not take into account the fact that proto-clusters are actually far more complex systems. For instance, the gas is not homogeneously distributed but may be mostly within dense small clouds. These clouds will suffer mass loss by ablation, thermal evaporation and photo-evaporation due to the large amount of ionizing photons emitted by the massive stars and by the SNe (e.g., Melioli $\&$ de Gouveia Dal Pino 2004, 2005). The diffuse gas will be heated by the $\mathrm{SNe}$, but may cool via radiative losses which depend on the square of the gas density, so that on one hand the SN remnant (SNR) shock fronts may cause the heating and expansion of the diffuse gas, but on the other hand, the mass loss from the clouds may increase the density of the diffuse gas enough to allow an efficient radiative cooling that may delay the gas removal. Defining the heating efficiency of the SNe, $\mathrm{HE}$, as the fraction of their energy that is effectively stored by the gas in the form of enthalpy and is not radiated away, then one may expect that an efficient gas expansion and removal will occur only when HE becomes close to unity.

In order to compute the evolution of the gas of a protocluster embedded in a SB environment considering the different physical processes mentioned above, we will adopt the semi-analytical dynamical model described by Melioli \& de Gouveia Dal Pino (2004, hereafter MGDP).

As in MGDP, we assume that an instantaneous burst of star formation occurs inside a spherical parent cloud of radius $R$. We adopt a Salpeter initial mass function (IMF) with a number of stars with mass greater than $8 M_{\odot}$ (i.e., the $\mathrm{SN}$ number) $\mathcal{N} \sim$ $0.01 M_{\text {star }}$, where $M_{\text {star }}$ is the total mass of stars in $M_{\odot}$.

For simplicity, it is assumed that the expanding SNRs and the cold, dense cloud clumps are uniformly distributed in the parent cloud. The clumps have an initial density $n_{\mathrm{c}} \sim 10^{4}$, radius $r_{\mathrm{c}} \sim 0.02 \mathrm{pc}$, and mass $M_{\mathrm{c}}=0.015 M_{\odot}$. Assuming similar characteristics to those observed in the Rosette giant cloud in our galaxy, in most of the calculations below we will adopt a total mass of clumps $M_{\mathrm{gas}} \simeq 8 M_{\mathrm{star}}$.

The equations that describe the evolution of the gas are given in MGDP. In the section below, we will present the evolution of the diffuse gas in a proto-cluster for different values of the SFE until the time when the heating efficiency, HE, which is mathematically defined as the ratio of the enthalpy flux through the boundary of the system to the total SN energy flux:

$\mathrm{HE}=4 \pi R^{2} \frac{\left(\frac{1}{2} \rho v^{2}+\frac{3}{2} p\right)}{\mathcal{R} \mathrm{E}_{\mathrm{SN}}} \mathcal{M} c_{\mathrm{s}}$,

becomes equal to unity.

\section{Results}

Table 1 summarizes the input parameters of the computed models. The values adopted are compatible with those estimated 

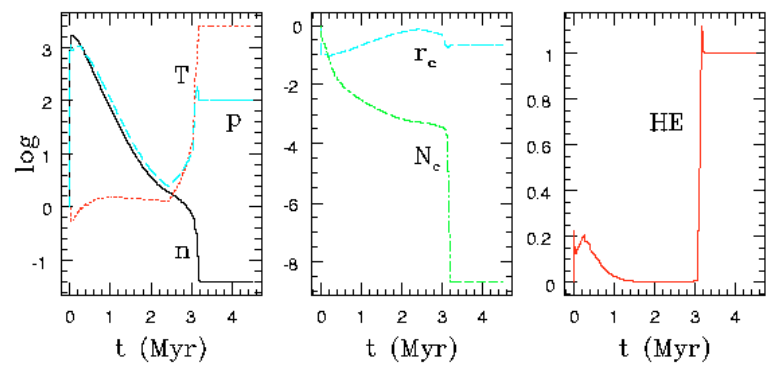

Fig. 1. Ambient density $(n)$, temperature $(T)$ and pressure $(p)$ evolution in the left panel for a spherical stellar cluster with initial $M_{\text {star }}=$ $10^{6} M_{\odot}, R=10 \mathrm{pc}$, ambient density $n=10 \mathrm{~cm}^{-3}, T=10^{4} \mathrm{~K}$, $p=1.38 \times 10^{-11} \mathrm{dy} \mathrm{cm}^{-2}$ and total gas mass $M_{\text {gas }}=8 \times 10^{6} M_{\odot}$. In the center panel: number of clouds $\left(N_{\mathrm{c}}\right)$ and clouds radius $\left(r_{\mathrm{c}}\right)$ evolution; and in the right panel: the SN heating efficiency (HE).

from the observations (e.g., Chandar et al. 2005; Fall 2004; Tenorio-Tagle et al. 2005, and references therein).

Figure 1 shows the time evolution of the ambient temperature, density, pressure, and the heating efficiency, HE, for the proto-cluster of model 1 of Table 1, for which $S F E=11 \%$. After $\sim 3 \mathrm{Myr}$, HE rapidly increases to one. Before that, the total energy stored in the gas is only a few percent of the total energy released by the $\mathrm{SNe}$, most of which is radiated away. During this period, the mass-loss rate from the clouds, mainly due to the process of photoevaporation (see MGDP), keeps the density of the diffuse gas high enough allowing an efficient radiative cooling. After this time, the clouds evaporated almost completely and the supernovae heating efficiency, $\mathrm{HE}$, increased to unity in less than $0.3 \mathrm{Myr}$ and this leads to a quick gas expansion and removal from the system. Note that right after $3 \mathrm{Myr}$ the gas temperature rises to $T \sim 10^{7} \mathrm{~K}$ and thus the gas will freely expand through the cluster radius in $t_{\text {exp }}=R / v_{\mathrm{s}}<0.1 \mathrm{Myr}$. These time scales are smaller than $\tau_{\text {cr }} \sim 0.5 \mathrm{Myr}$ so that, once HE reaches the unity value, all the gas will be removed from the cluster in less than one cluster dynamical crossing time and, since $\sigma \geq v_{\text {esc }}$, or $S F E \leq 50 \%$, we may conclude that the system will become unbound and disperse.

Figure 2a compares HE for systems with different initial radius. As expected, the larger the radius of the proto-cluster the longer the system will take to reach a heating efficiency equal to unity, but in all cases we observe the same trend as in Fig. 1, i.e., a rapid change in HE from $\ll 1$ to $\sim 1$ in less than the corresponding $\tau_{\mathrm{cr}} \sim 0.5,1.4$, and $2.6 \mathrm{Myr}$, for the systems with $R=$ 10,20 , and $30 \mathrm{pc}$, respectively. Figure $2 \mathrm{~b}$ shows the evolution of HE for different SFEs. All the curves have the same initial conditions of Fig. 1 except for the initial total gas mass $\left(M_{\mathrm{gas}}\right)$. We see that the higher the SFE (i.e., the smaller the total gas content of the system relative to the total amount of mass in stars) more rapidly the system reaches a high HE and therefore, earlier is the gas removal. Also, since for the investigated range of SFE values, $S F E=10 \%-50 \%$, the timescales for gas removal right after $\mathrm{HE}$ reaches the unity value are smaller than the clusters dynamical crossing times, $\tau_{\mathrm{cr}} \sim 0.5,0.8,1 \mathrm{Myr}$, for the clusters with $S F E=11 \%, 30 \%$, and $50 \%$, respectively, then they all should become gravitationally unbound systems.

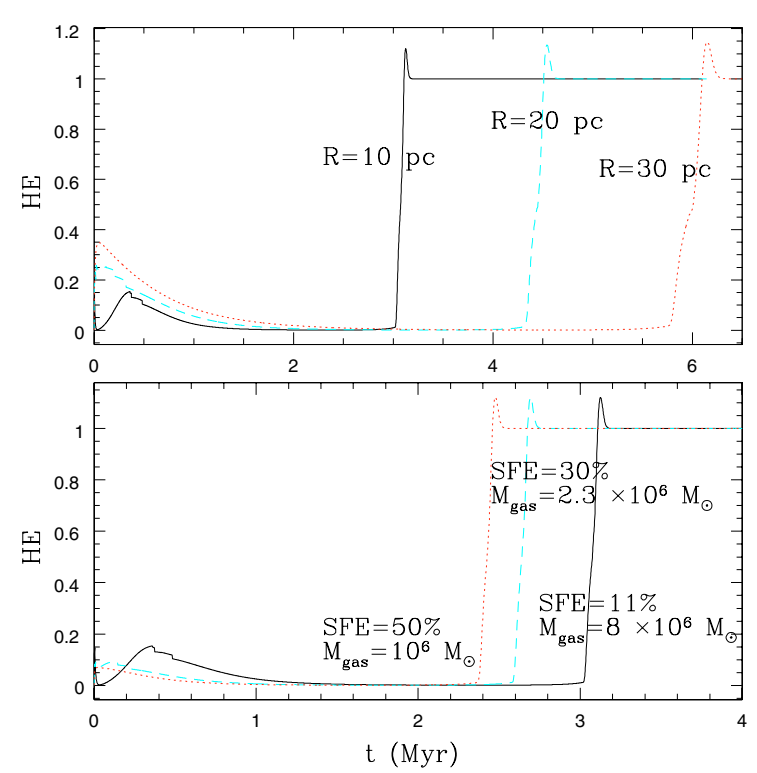

Fig. 2. a) Top: HE computed for different initial radius. All the curves have the same initial conditions of Model 1 of Fig. 1 (with a $S F E=$ $11 \%$ ), except for the radius of the system. $R=10 \mathrm{pc}$ is represented by the dot-dashed lines, $R=20 \mathrm{pc}$ by solid lines, and $R=30 \mathrm{pc}$ by dashed lines. b) Bottom: evolution of HE for different SFEs. All the curves have the same initial conditions of Model 1 except for the initial gas mass content. $S F E=11 \%$ (with $M_{\text {gas }}=8 \times 10^{6} M_{\odot}$ ) is represented by the dot-dashed lines, $S F E=30 \%$ (with $M_{\text {gas }}=2.3 \times 10^{6} M_{\odot}$ ) by solid lines, and $S F E=50 \%$ (with $M_{\mathrm{gas}}=10^{6} M_{\odot}$ ) by dashed lines.

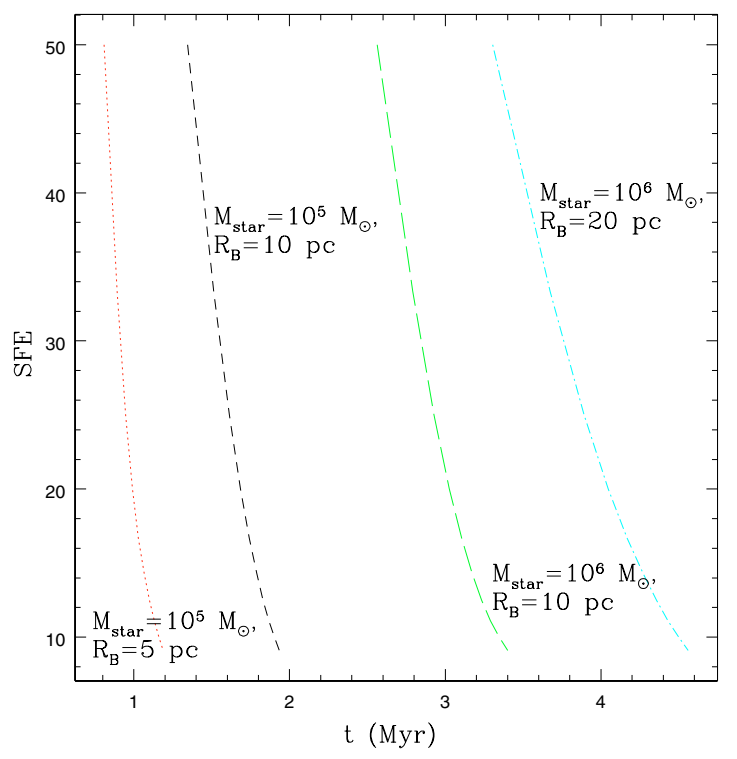

Fig. 3. SFE versus the time $\tau_{\mathrm{HE}}$ that the system takes to reach $\mathrm{HE}=1$ for clusters with different initial conditions (Models 1 to 4 of Table 1).

Figure 3 adds information to the results of Fig. 2b, showing SFE versus the time $\tau_{\mathrm{HE}}$ that the system takes to reach $H E=1$ for clusters with different initial conditions (Models 1 to 4 of Table 1). $\tau_{\mathrm{HE}}$ can be also interpreted as the time the gas is retained in the proto-cluster possibly allowing for new generations of star formation. The curves indicate that this timescale in a cluster is very insensitive to SFE when the total initial gas 
mass content is fixed. $\tau_{\mathrm{HE}}$ varies less than $30 \%$ between $S F E=$ 0.1 and 0.5 in each example. Combined with the results of the previous figures, Fig. 3 also reveals that due to a quick gas removal right after HE becomes equal to one, typical SB protoclusters are expected to start to disperse after $\leq 5 \mathrm{Myr}$.

\section{Conclusions}

We have investigated the evolution of the gas in proto-clusters embedded in SB systems and have shown that even in the presence of dense, cold clouds that tend to inhibit the heating of the interstellar gas by the SNE, the timescales during which the gas is retained in these systems do not exceed $\sim 5 \mathrm{Myr}$ (considering proto-clusters with total mass in the range $M_{\text {star }}+M_{\text {gas }} \sim 6 \times$ $10^{6}-6 \times 10^{7} M_{\odot}$ and radii $\left.5 \mathrm{pc} \leq R \leq 20 \mathrm{pc}\right)$. We have also found that after this time the gas is removed very rapidly $(\sim 0.5 \mathrm{Myr})$ in timescales smaller than the clusters dynamical crossing time and this result is approximately insensitive to the initial SFE for a given total gas mass. These very short timescales for gas removal combined with values of $S F E \leq$ $50 \%$ will make these systems to start to disperse after $\sim 5 \mathrm{Myr}$.
Acknowledgements. C.M. and E.M.G.D.P acknowledge financial support from the Brazilian Agencies FAPESP and CNPq.

\section{References}

Chandar, R., Leitherer, C., Tremonti, C. A., et al. 2005, ApJ, 628, 210

Fall, S. M. 2004, ASPC, 322, 399

Lada, C. J., \& Lada, E. A. 2003, ARA\&A, 41, 57

Melioli, C., \& de Gouveia Dal Pino, E. M. 2004, A\&A, 424, 817 (MGDP)

Melioli, C., de Gouveia Dal Pino, E. M., \& Raga, A. 2005, A\&A, 443, 495

Meurer, G. R., Heckman, T. M., Leitherer, C., et al. 1995, ApJ, 110, 2665

Tenorio-Tagle, G., Silich, S., Rodríguez-González, A., \& Munoz-Tunon, C. 2005, ApJ, 628, 13

Tremonti, C. A., Calzetti, D., Leitherer, C., \& Heckman, T. M. 2001, ApJ, 555, 322

Whitmore, B. C., Gilmore, D., Leitherer, C., et al. 2005 [arXiv: astro-ph/0507706] 